\title{
EpiTEome: Simultaneous detection of transposable element insertion sites and their DNA methylation levels
}

\author{
Josquin Daron and R. Keith Slotkin *1)
}

\begin{abstract}
The genome-wide investigation of DNA methylation levels has been limited to reference transposable element positions. The methylation analysis of non-reference and mobile transposable elements has only recently been performed, but required both genome resequencing and MethylC-seq datasets. We have created epiTEome, a program that detects both new transposable element insertion sites and their methylation states from a single MethylC-seq dataset. EpiTEome outperforms other split-read insertion site detection programs, even while functioning on bisulfite-converted reads. EpiTEome characterizes the previously discarded fraction of DNA methylation at sites of new insertions, enabling future investigation into the epigenetic regulation of non-reference and transposed elements.
\end{abstract}

Keywords: Methylome, Transposable elements, Insertion site, Bisulfite, Split reads, Bioinformatics, MethylC-seq

\section{Background}

Transposable elements (TEs) are mobile fragments of DNA that inhabit the nuclear genome of all eukaryotes. TEs are dynamic, insert into chromosomal loci, generate polymorphisms, and create mutations in protein-coding genes [1, 2]. TE sequences have diversified and are classified by families with common sequences, domains, structures, and mobilization strategies [3, 4]. Some TE families have been evolutionarily successful, going through bursts of activity [5], which result in the expansion and rearrangement of genomes [6,7]. Due to their ability to create rearrangements and mutations, TEs are targeted for epigenetic silencing. Multiple overlapping mechanisms recognize TE sequences and modify their chromatin with DNA and histone methylation, resulting in the formation of heterochromatin that lacks proteincoding expression (reviewed in [8]).

5-Methylcytosine is a DNA modification targeted to TEs to inhibit their transcriptional activity. DNA methylation is originally targeted to cytosines $(\mathrm{Cs})$ in any sequence context by the activity of small RNA-directed DNA methylation (RdDM) (reviewed in [8]). Once established

\footnotetext{
* Correspondence: Slotkin.2@osu.edu

Department of Molecular Genetics, The Ohio State University, Columbus, OH, USA
}

in plants and vertebrates, DNA methylation can be copied and epigenetically maintained at the TE by the activity of methyltransferase proteins. In plants, DNA methylation is maintained at different levels by distinct methyltransferase proteins in the CG, CHG, and $\mathrm{CHH}$ sequence contexts (where $\mathrm{H}=\mathrm{A}, \mathrm{C}$, or $\mathrm{T}$ ). DNA methylation is detected by bisulfite sequencing, where non-methylated cytosines are chemically converted and appear as thymine via DNA sequencing, while methylated cytosines are not converted. Bisulfite-conversion whole-genome sequencing library amplification and sequencing [9]. The standard of MethylC-seq data analysis involves mapping ylC-seq reads to a reference genome. Consequently, positions are overlooked.

The detection of non-reference and mobile TE insertion sites has been traditionally performed individually semble each one, identifying polymorphic TE insertion sites. However, short read-length, genome complexity, 
and high cost prohibit this strategy. Alternatively, genome resequencing and alignment to an available assembled reference genome sequence has been used to detect TE insertion sites. This is accomplished by focusing on the sequencing reads that do not match the reference genome using a split-read mapping strategy to align one end of a single read to the reference genome, while the other half of the read maps to a known TE end [11]. Two such programs are SPLITREADER and TEPID, which have successfully detected TE insertion sites across the resequencing of 216 Arabidopsis natural ecotypes, identifying evolutionarily active TE copies and transposition hotspots $[12,13]$. These new insertions sites are generally excluded from the genome-wide analysis of DNA methylation by MethylC-seq. Only recently has the genome-wide DNA methylation of new TE insertion sites been assayed; however, this required both whole-genome resequencing and MethylC-seq datasets [12].

We aimed to utilize the vast and available MethylCseq data the epigenomics community generates to identify new TE insertion sites rather than resequencing these genomes. We have combined the fields of DNA methylation and TE insertion site detection by creating a program called epiTEome. This program can for the first time identify a TE insertion from MethylC-seq reads, as well as determine the methylation state of the new TE insertion and surrounding insertion site. This program circumvents the necessity to perform genome resequencing to identify new $\mathrm{TE}$ insertion sites, reducing the required cost to analyze TE insertion site and DNA methylation data to a single MethylC-seq experiment.

\section{Results}

\section{Description of the epiTEome program}

Unlike other programs developed to identify new TE insertion sites, epiTEome was developed to initiate analysis with MethylC-seq reads generated from whole-genome sequencing of bisulfite-converted DNA. Before mapping, reads are trimmed and processed to remove adapters, low quality and imperfect sequencing reads from a FASTQ file (preprocessing, Fig. 1a). The trimmed and filtered reads are then mapped to the reference genome using Bismark [14] or any MethylC-seq mapping program. Stringent filtering and sensitive mapping are suggested to reduce the fraction of low quality unmapped reads, as the MethylC-seq reads that fail to map to the reference genome are the input to epiTEome (Fig. 1a).

EpiTEome splits and maps each MethylC-seq read that failed to align to the reference genome. The initial length of each spit-read end is user-defined; however, it should be over 25 nucleotides (nt). EpiTEome first identifies the reads with discordant (map to different locations in the genome) ends using the mapping program Segemehl [15] (operation, Fig. 1a). Once the discordant reads are identified, the corresponding full-length read is split into all possible combinations with a minimal length of $25 \mathrm{nt}$. Each variation of the split-read is mapped to the reference genome to identify the breakpoint location on the read where one half maps to a TE and the other half to the new insertion site (Fig. 1b). This process identifies the point of the read that transitions from one discordant position to another and only the read split at this position is retained for analysis of TE insertion sites (Fig. 1c) and DNA methylation (Fig. 1d).

Discordant split reads are processed by filtering for those with at least one end at the edge of an annotated TE (operation, Fig. 1a). If both ends discordantly map to the same TE family (likely due to frequent TE internal deletions), the read is discarded. Discordant reads are next clustered based on their location in the genome and further filtered. Read clusters are filtered for: (1) the number of split-reads supporting the new insertion site $(>5)$; (2) both ends of the same TE must be represented at the insertion site; and (3) the overlap of the reads at the insertion site should not extend beyond the target site duplication (TSD) generated by TE insertion (Fig. 1c). EpiTEome results are reported as coordinate positions of each TE insertion site, TE family, and parental TE copy (reporting, Fig. 1a). Application of this workflow identifies sites of new TE insertion, the TE TSD, and mobile TE families (analysis, Fig. 1a).

To this point in the workflow, epiTEome functions similarly to SPLITREADER and TEPID to identify new TE insertion sites (Fig. 1b). The added value of epiTEome is the ability to detect the DNA methylation status of the transposed TE and insertion site using the exact split-reads that identified the transposition event. DNA methylation is reported as single-insertion alignments (Fig. 1d), as well as meta-analysis of all insertion sites in the sample. The methylation is split into CG, CHG, and $\mathrm{CHH}$ contexts for both the $5^{\prime}$ and $3^{\prime}$ (non-TE) flank of the insertion site (Fig. 1d), as well as the $5^{\prime}$ and $3^{\prime}$ TE ends (analysis, Fig. 1a).

\section{Detection of simulated TE insertions}

To test the sensitivity (true positive [TP] rate) of epiTEome, we created 84 synthetic (simulated) TE insertions into Arabidopsis chromosome 2. We chose a plant genome because plants methylate cytosines in all sequence contexts (CG, CHG, $\mathrm{CHH}$ ), producing a computational challenge in MethylC-seq analysis. Synthetic insertions represented the seven types of mobile TE families previously identified in genome-wide transposition experiments across the Arabidopsis thaliana species range: Copia elements (ATCOPIA78/Onsen and ATCOPIA93/Evadé), Gypsy elements (ATGP2 and ATHILA2), AtEnSPM elements (ATENSPM5 and ATENSPM6), Mutator elements (VANDAL6 and BOMZH1), Helitron elements (ATREP2A and ATREP11A), hAT elements (SIMPLEHAT2 and ATHATN2), and the 


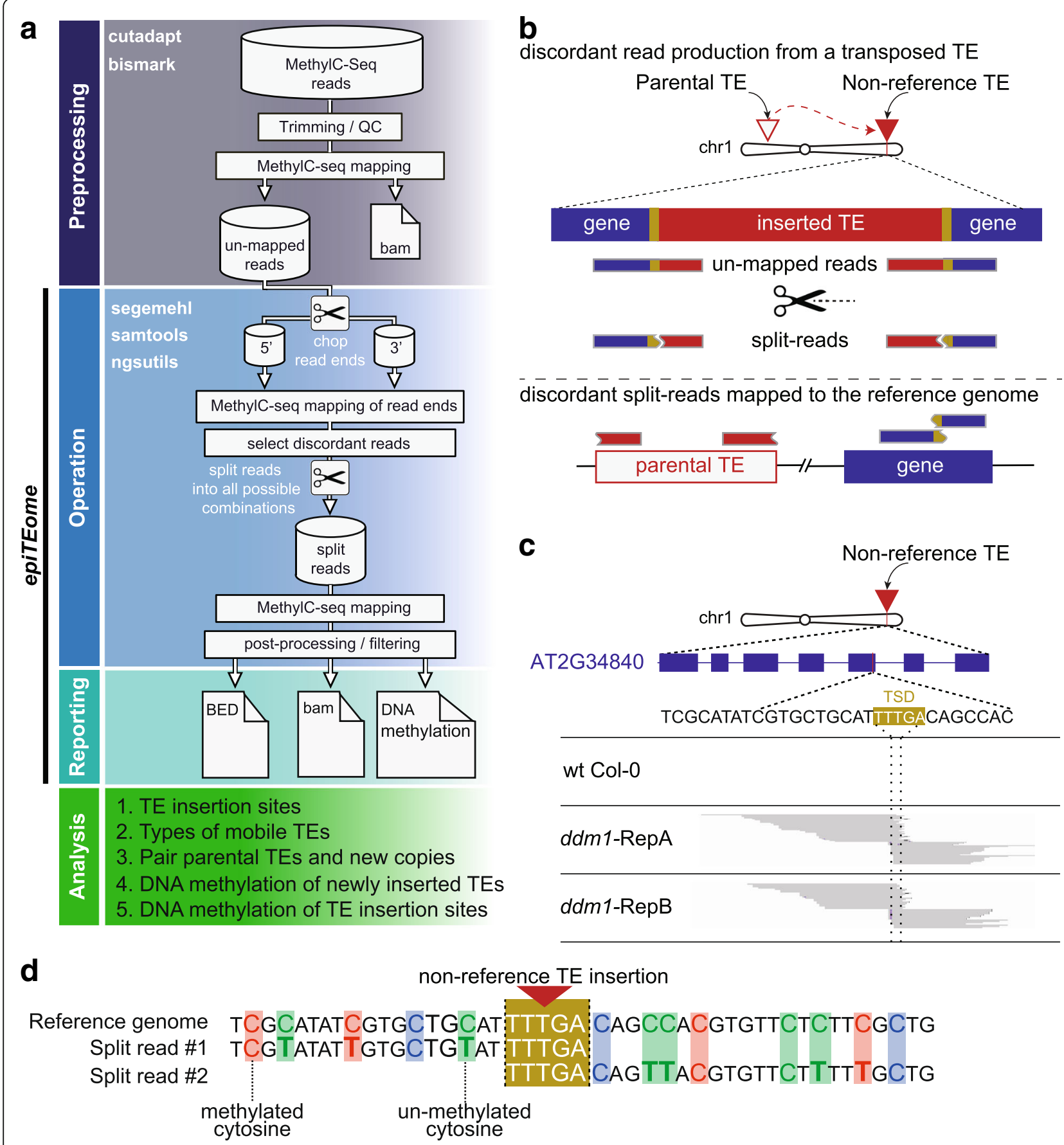

Fig. 1 Design of epiTEome function. a Workflow of methodology developed to identify non-reference insertions of TEs using filtered MethylC-seq reads that fail to align to the reference genome. $\mathbf{b}$ Principle behind split-read detection of new TE insertion sites. Reads that fail to fully map to the reference genome are used to identify the sites of new TE insertion. Non-mapping reads are split and mapped to the reference genome to identify reads with one end that maps to a TE and the other end to the site of insertion. c Example of a new TE insertion detected by epiTEome in Arabidopsis: $d d m 1$ mutants undergo TE transcriptional reactivation and transposition [30]. Split reads not present in wild-type (wt Col-0) identify a TE insertion into the gene At2g34840 in two biological replicates of ddm1 MethylC-seq (RepA and RepB). The 5' and 3' flanking spit reads overlap (dashed lines) at the target site duplication (gold sequence) generated by TE insertion. $\mathbf{d}$ In addition to identifying new TE insertion sites, epiTEome detects the cytosine methylation status at these loci. Sequence alignment of split MethylC-seq reads at the insertion site are used to determine the cytosine DNA methylation status. Unconverted cytosines represent methylated bases, while $\mathrm{C} \rightarrow \mathrm{T}$ transitions (bold) in the MethylC-seq reads represent unmethylated cytosines. The sequence context of each cytosine is displayed (CG $=$ red, $\mathrm{CHG}=b / \mathrm{b}, \mathrm{CHH}=$ green) 
LINE element (ATLINE2) [12, 13]. We created simulated insertions into the chromosomal contexts of genes, TEs, and non-TE/non-gene "intergenic" regions. We tested SPLITREADER and TEPID using in silico generated DNA sequencing reads and epiTEome on the same dataset that we in silico bisulfite-converted assuming the genome-wide rates of CG and $\mathrm{CH}$ methylation (see "Methods"). We found that TE insertions were detected at $92 \%$ sensitivity by epiTEome using bisulfite-converted reads, a higher sensitivity compared with programs that do not use bisulfiteconverted reads (Fig. 2a). For insertions into repetitive regions, reduced sensitivity occurs for each of the programs tested, including a drop from $95 \%$ (insertion into a gene) to 86\% (insertion into a TE) sensitivity for epiTEome (Fig. 2a). However, epiTEome remains the most sensitive TE insertion site detection program independent of TE insertion site (Fig. 2a), even while using bisulfite-converted MethylCseq reads. We also calculated the false discovery rate (FDR) of epiTEome (3.26\%) and demonstrate that it is comparable to SPLITREADER and TEPID (Fig. 2b). We therefore conclude that epiTEome is a comparably sensitive and accurate detector of TE insertion sites, while using the distinct MethylC-seq data source.

To determine the factors responsible for accurate TE insertion detection from MethylC-seq data by epiTEome, we tested epiTEome sensitivity while altering four parameters: sequencing depth; read length; methylation level; and number of non-bisulfite conversion-induced single nucleotide polymorphisms (SNPs). We in silico distributed 84 TE copies randomly on Arabidopsis chromosome 2 and independently tested each of the four variables (see "Methods"). We found that the level of methylation and read length are not critical, as long as the read length is over $75 \mathrm{nt}$ (post-trimming) (Fig. 2c). More critical are the depth of sequencing/genome coverage and the number of (non-bisulfite-induced) SNPs present between the reads and reference sequence. Therefore, as a best practice to identify TE insertions from MethylC-seq data, we suggest sequencing using $\geq 100$ nt length reads, depth of $\geq 18 x$, while using a closely related reference genome.

\section{EpiTEome accurately detects TE insertions from MethylC-seq data}

To determine if epiTEome can detect TE insertions from biologically relevant MethylC-seq data, we investigated naturally occurring Arabidopsis ecotypes because these samples have been subjected to both genome resequencing and MethylC-seq analyses [16-18]. We chose the Ha- 0 and Rou- 0 closely related ecotypes because both SPLITREADER and TEPID have previously identified unique insertions in Ha-0 compared with Rou- 0 and the reference Col-0 ecotype based on genome resequencing data $[12,13]$. EpiTEome was launched on publicly available MethylC-seq reads from $\mathrm{Ha}-0$ (85 nt reads, $21 \mathrm{x}$ coverage, $0.31 \mathrm{SNPs} / \mathrm{read}$ compared to the Col-0 reference calculated in [18]) [17]. The overlap between identified Ha- 0 TE insertions between all three programs is
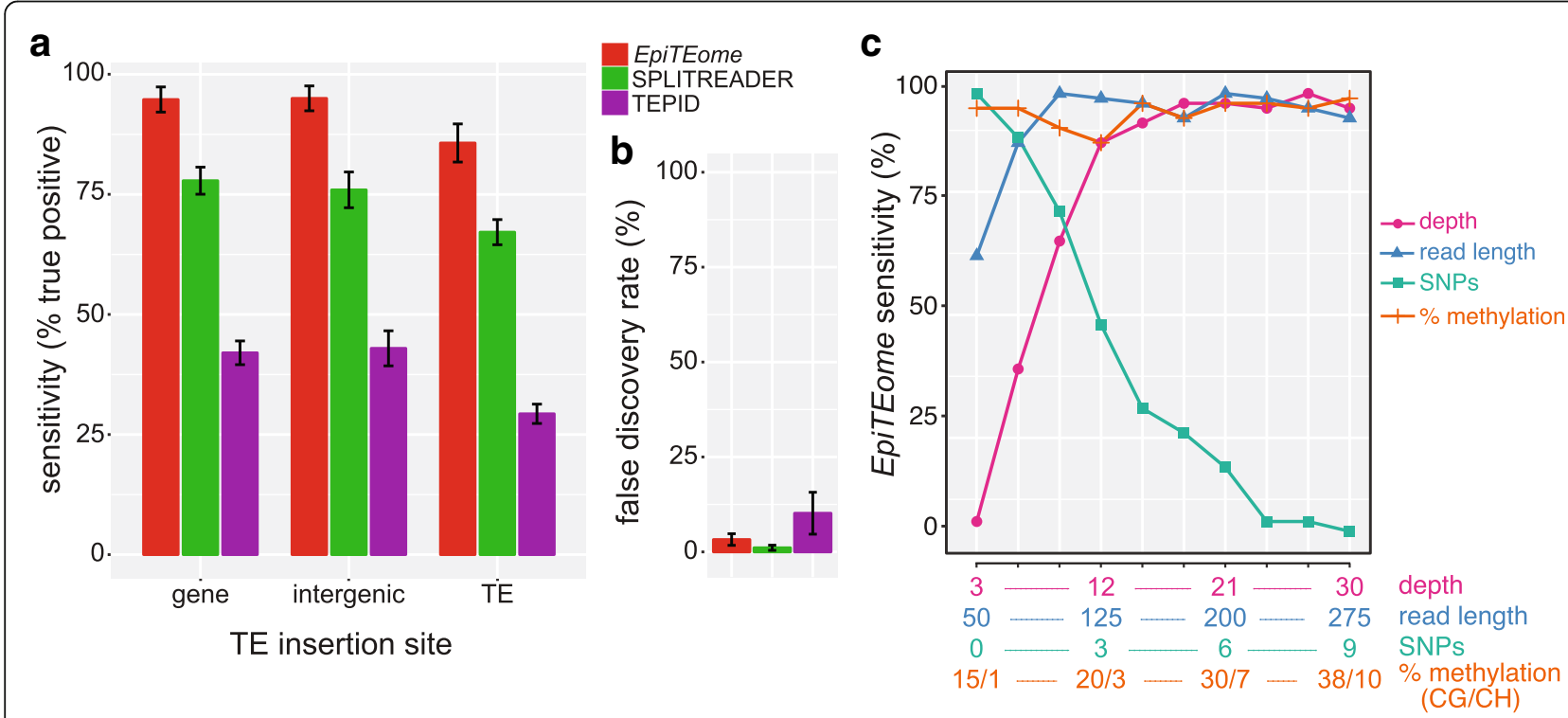

Fig. 2 Validation of epiTEome on simulated data. a Bar plot of sensitivity of detection for simulated TE insertions at three different TE insertion contexts (gene, intergenic, TE). SPLITREADER and TEPID use non-bisulfite converted reads, while epiTEome utilizes bisulfite-converted MethylC-seq reads. b FDR of epiTEome, SPLITREADER, and TEPID calculated from the same simulated data as part A. Error bars in (a) and (b) represent the 95\% confidence interval (Cl) generated using five replicates. c Analysis of how the variables of sequencing depth, read length, methylation level, and number of SNPs affect epiTEome sensitivity. Throughout the analysis in (c), epiTEome produced a $2.88 \%$ false-positive average, with a standard deviation of 1.45 



Fig. 3 Validation of epiTEome using published MethylC-seq data. a Venn diagram comparing three independent programs created to identify TE insertion sites in the Arabidopsis ecotype Ha-0. EpiTEome is the only program that utilizes MethylC-seq data. Color codes are maintained throughout panels (b)-(d). Split-reads identify the insertion and target site duplication of a TE insertion detected by all three programs (b) and a TE insertion specifically detected by epiTEome (c). The split-read analysis is confirmed by the decrease in coverage of un-split MethylC-seq reads in Ha-0 (insertion present) vs. the ecotype Rou-0 (insertion absent). d Meta-plot of MethylC-seq un-split read coverage at the TE insertion sites and flanking regions uniquely detected by each program or detected by all three. e MethylC-seq un-split read coverage z-score for each of the $175 \mathrm{TE}$ insertions uniquely identified by epiTEome, plus the 16 detected by all three programs (asterisks). Seven percent of the insertion sites with high un-split read coverage (bracket) at the TE insertion site are likely false positives (FP)

shown in Fig. 3a. Sixteen TE insertions were identified by all programs (black), including the ATCOPIA93 (Evadé) insertion into At1g09930 (creating the target site duplication CTTGC) shown in Fig. 3b. Therefore, epiTEome can successfully detect known TE insertion sites from the unique MethylC-seq data source.

Although epiTEome, SPLITREADER, and TEPID identified some non-reference TE insertion sites in common, many insertions were uniquely identified by only one program (Fig. 3a). To determine if a detected TE insertion site is real, we performed a verification analysis based on the mapping coverage of the un-split reads. For example, due to the TE insertion into At1g09930 in the Ha-0 ecotype, un-split read coverage at this site is absent compared with the flanking DNA. This lack of un-split read coverage is due to all of the reads from this region having TE content and thus not mapping the reference genome that lacks the TE insertion (Fig. 3b). This is in contrast to the full unsplit read coverage of the closely related ecotype Rou- 0 that does not carry the TE insertion (Fig. 3b). TE insertions detected by split-reads can therefore be confirmed by a reduced coverage of un-split reads at the insertion site compared to the flanking DNA.

We next investigated a TE insertion site only detected by epiTEome. An ATCOPIA58 insertion into chromosome 4 was detected in the MethylC-seq split reads by epiTEome and is verified by the lack of un-split read coverage at the insertion site (compared to Rou-0 which does not have the TE insertion) (Fig. 3c). To determine if a majority of the 175 TE insertions identified only by epiTEome (Fig. 3a) are true positives, we performed a meta-analysis of the un-split MethylC-seq read coverage of TE insertion sites that were detected by all programs (black), or uniquely by epiTEome (red), TEPID (purple) or SPLITREADER (green) (Fig. 3d). We find the highest confidence in the TE insertions detected by all three programs, while the unique TE insertions detected by epiTEome (from MethylC-seq) are supported by decreased un-split read coverage at the insertion site. 
The 12 TE insertion sites uniquely detected by SPLITREADER are likely missed by epiTEome (false negatives [FNs]), while the 247 insertions uniquely identified by TEPID are not supported in the MethylC-seq data. These unconfirmed insertions detected by TEPID are likely not due to the accuracy of the program, but rather due to the fact that TEPID TE insertions are identified based on genome resequencing, not the MethylC-seq dataset that we are testing it against. To further determine the rate at which epiTEome was detecting FPs, we investigated the un-split MethylCseq read coverage of each of the TE insertion sites uniquely detected by epiTEome or detected by all three programs. This analysis demonstrates that a decrease in un-split read coverage occurs for 93\% of these TE insertion sites (Fig. 3e), providing confidence that the majority of TE insertions detected by only epiTEome are true positives from the MethylC-seq raw data.

\section{Detection of new TE insertions in repetitive crop genomes}

To determine if epiTEome could function to identify TE insertions within larger and more complex plant crop genomes, we performed a simulated detection of new TE insertions in maize (2.3 Gbp, 85\% TEs [19]) and rice (389 Mbp, $35 \%$ TEs [20]). As expected, epiTEome functions with the highest sensitivity on low copy number TEs inserted into genic regions (Fig. 4a, b). When the retrotransposon copy number exceeds 100, and when inserted into TE regions of the genome, the sensitivity of epiTEome drops to $25 \%$ in maize and $56 \%$ in rice (Fig. 4a, b). This sensitivity drop represents the computationally most difficult TE insertions to detect and these TE-into-TE insertion events are associated with an increase in FDR (Fig. 4c). This suggests that epiTEome will function better for less repetitive genomes or when performed on highly repetitive genomes such as maize epiTEome will be most sensitive to detect insertion of DNA TEs into genes.

To test epiTEome on biological data, we investigated published maize MethylC-seq data to identify TE insertion variation in the standard inbred line Oh43 compared with the reference genome strain B73. We chose Oh43 because it represents a computational challenge due to the large variation (non-bisulfite-induced SNPs) between maize inbred lines. In addition, the Oh43 MethylC-seq had not
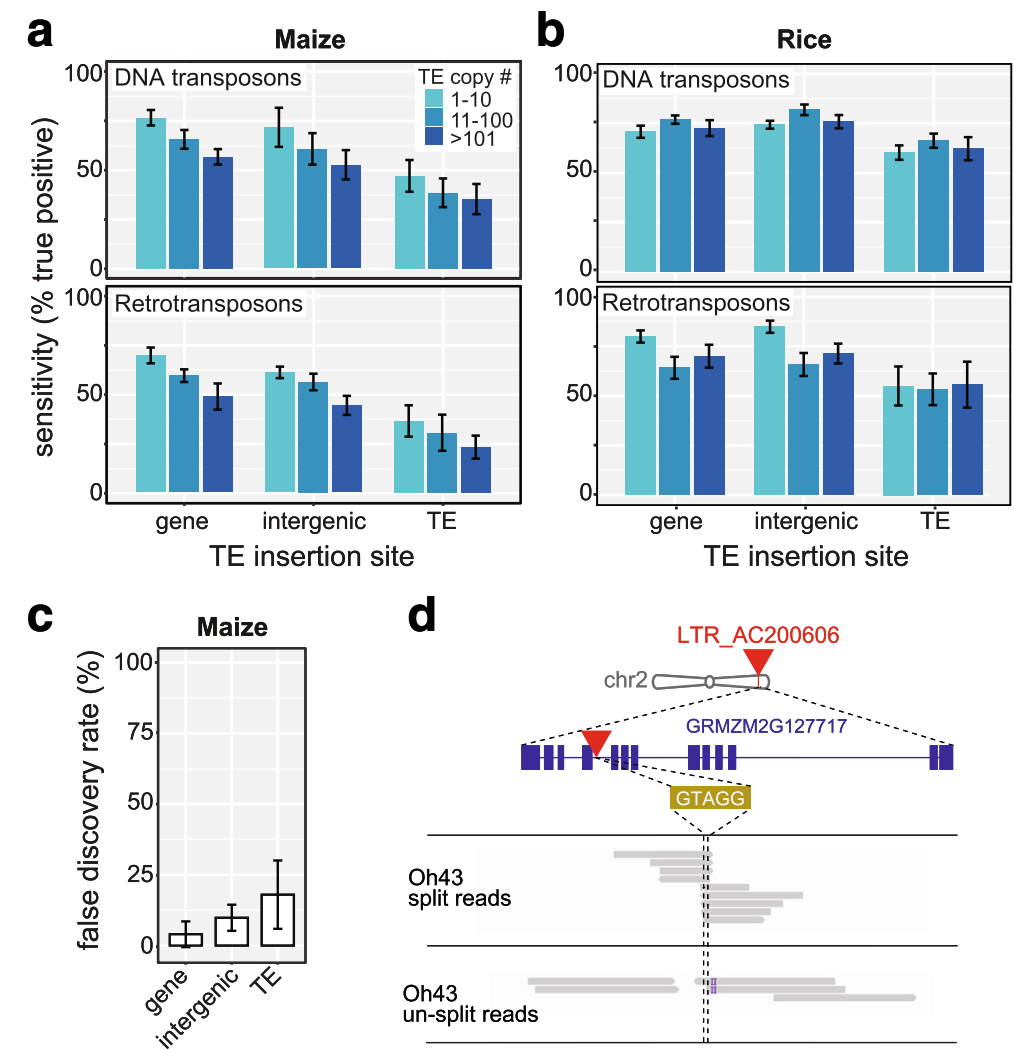

Fig. 4 EpiTEome detects new TE insertions in repetitive crop genomes. $\mathbf{a}$, b Bar plot of sensitivity of detection for simulated TE insertions at three different potential TE insertion contexts (gene, intergenic, TE) in the maize (a) or rice (b) genomes using in silico generated bisulfite-converted reads. Results are divided by TE copy number. c FDR of epiTEome calculated from the same simulated data as part A. Error bars in (a)-(c) represent the $95 \% \mathrm{Cl}$ generated using five replicates. $\mathbf{d}$ Genome browser visualization of a non-reference (not in the reference B73 genome) LTR retrotransposon TE insertion into the PFK5 GRMZM2G127717 gene in the Oh43 inbred line identified by epiTEome 
been subjected to target enrichment via sequence capture, but rather represented the whole genome at 13x coverage [21]. We found $18 \mathrm{TE}$ insertions (eight into genes, Table 1) in Oh43 compared with B73. For example, we identified an LTR retrotransposon TE inserted into an intron of the PFK5 protein-coding gene, which was verified as a gap in un-split read coverage at this site (Fig. 4d). This analysis demonstrates that epiTEome is capable of detecting TE insertions from MethylC-seq datasets produced from repetitive crop genomes.

\section{EpiTEome identifies the cytosine methylation status of non-reference TEs and flanking DNA}

To demonstrate epiTEome's ability to capture and report the DNA methylation state of TE insertion sites, we used the Arabidopsis Ha-0 ecotype and calculated the average DNA methylation at: (1) each non-reference TE insertion; (2) the flanking non-TE DNA of the insertion site; (3) these same loci without the insertion in the reference Col-0; and (4) the parental TEs that produced the transposed copies in Ha-0 (Fig. 5). We confirmed that transposition of a TE recruits DNA methylation to the insertion site (Fig. 5, middle), which without TE insertion have low DNA methylation levels (Fig. 5, top) [12, 22]. These non-reference TEs and flanking DNA have similar DNA methylation levels compared with their parental TE loci (Fig. 5, bottom). Of note, we observed higher $\mathrm{CHH}$ methylation levels at the more recently inserted TEs and flanking sites compared to the parental TE copies, suggesting that the more recent copies are more efficient targets of small RNA-directed DNA methylation. Using a MethylC-seq read of $85 \mathrm{nt}$, we were able to determine DNA methylation states of $60 \mathrm{bp}$ on either side of the TE insertion site (Fig. 5, right side). This window of resolution will increase with longer sequencing reads; however, it is long enough to detect a difference between DNA methylation contexts at the TE insertion sites: while CG and CHG methylation linearly decrease from the flanks of a more recent TE insertion site,
$\mathrm{CHH}$ methylation exponentially decreases to a low level within 40 bases of the insertion site (Fig. 5, middle right).

\section{Discussion}

EpiTEome represents an improvement both in the sensitivity of TE insertion site detection (Fig. 2a), as well as the ability to use MethylC-seq data. However, there are technical and biological limitations that must be imposed on epiTEome to reduce the number of FPs. For example, sequencing depth greatly impacts epiTEome sensitivity (Fig. 2c). Increased sequencing depth increases the likelihood of reads with discordant breakpoint junctions near their center. Due to the reduced complexity of the DNA code using bisulfite-converted DNA, the number of non-bisulfite-induced SNPs between the reads and reference genome also significantly alters epiTEome sensitivity (Fig. 2c). This will limit TE insertion site detection in lines without a closely related reference genome and will work better for investigation of tissues or mutants within one strain, rather than between multiple strains.

In addition to mapping limitations, epiTEome filtering steps remove TE insertion sites within a TE of the same family. This limits insertion detection for TEs that both compose and target heterochromatin, such as some LTR retrotransposons, which are often inserted into similar TE copies [23]. This filtration was necessary to avoid the split-read detection of frequent TE internal deletions produced by non-homologous recombination (not real transposition events). EpiTEome will also be more sensitive to detect germinal transposition events rather than somatic. Germinal events will be present in all of the sampled DNA and thus appear in multiple reads along with a correlation of decreased un-split read coverage at that TE insertion site. Somatic insertion split-reads may be rare (depending on the timing/size of the sector) and likely will not have an observable decrease of un-split read coverage.

Table 1 EpiTEome-identified non-reference TE insertions in maize Oh43 genes compared to the B73 reference

\begin{tabular}{|c|c|c|c|c|c|c|c|}
\hline Gene ID & Gene annotation & Chromosome & Insertion site & Genic location & TE family & TE superfamily & $\begin{array}{l}\text { Supporting } \\
\text { spit reads }\end{array}$ \\
\hline GRMZM2G127717 & Phosphofructokinase 5 (PFK5) & 2 & 181762712 & Intron & LTR_AC200606 & Unknown LTR & 9 \\
\hline GRMZM2G343360 & $\begin{array}{l}\text { SAUR-like auxin-responsive } \\
\text { protein family }\end{array}$ & 2 & 197668412 & Exon & LTR_AC191075 & Unknown LTR & 5 \\
\hline GRMZM2G014994 & GPI-anchored protein & 4 & 163300669 & Intron & Gypsy-173_ZM-LTR & Gypsy LTR & 7 \\
\hline GRMZM2G178753 & Kinase-like protein & 5 & 19547332 & Exon & Gypsy-143_ZM-LTR & Gypsy LTR & 5 \\
\hline GRMZM2G330684 & Ring Zinc Finger & 5 & 30857925 & Intron & HARB-N3_ZM & Harbinger & 5 \\
\hline GRMZM2G148229 & $\begin{array}{l}\text { SNARE associated Golgi } \\
\text { protein family }\end{array}$ & 7 & 170575334 & Intron & GYZMA1_LTR & Gypsy LTR & 5 \\
\hline GRMZM5G871262 & LIMR family protein & 9 & 24960353 & Intron & Gypsy29-ZM_LTR & Gypsy LTR & 7 \\
\hline GRMZM2G111066 & Protein SIP5 isoform X1 & 9 & 105921345 & exon & Gypsy29-ZM_LTR & Gypsy LTR & 6 \\
\hline
\end{tabular}



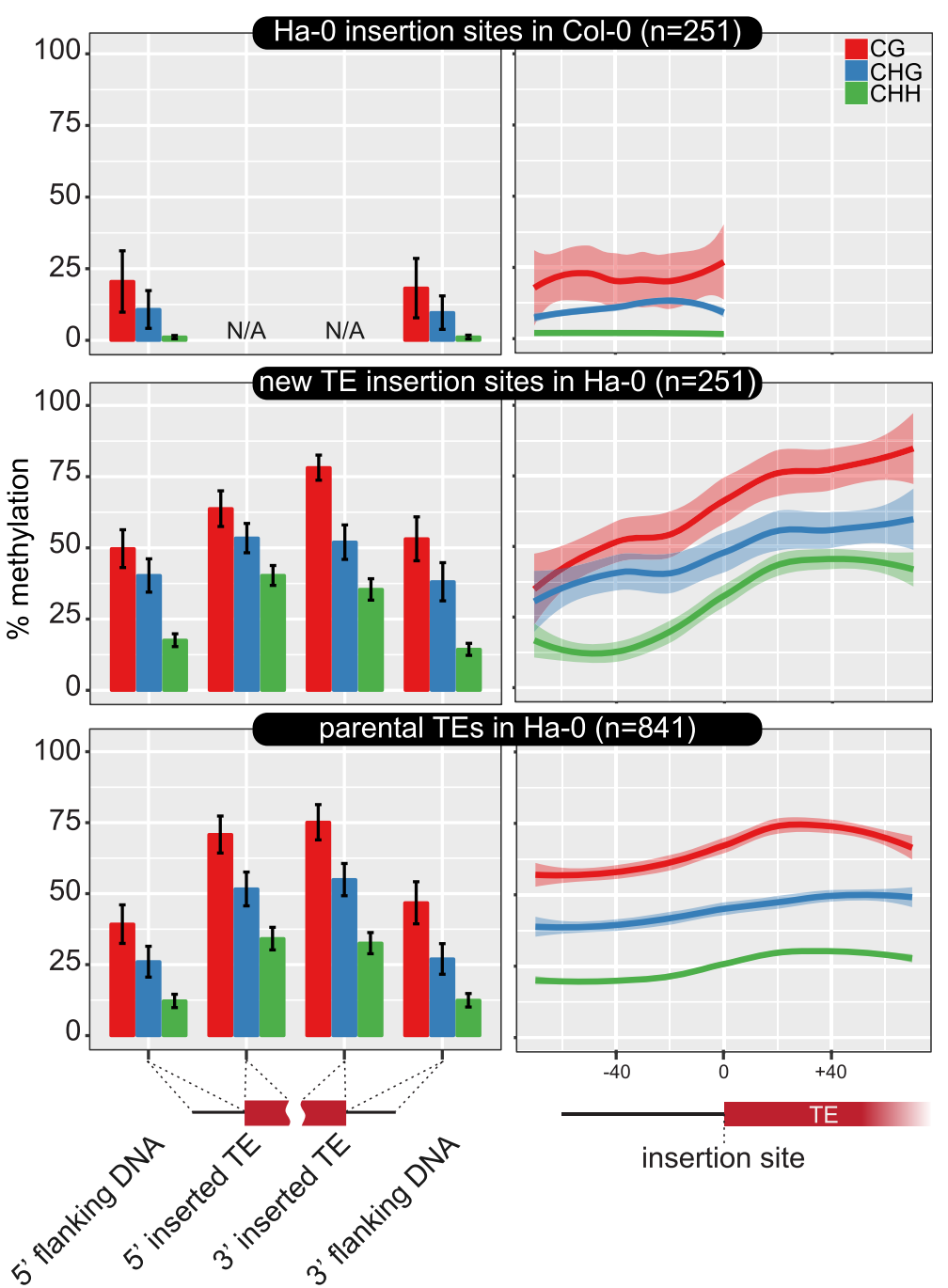

Fig. 5 EpiTEome detects DNA methylation at new TE insertion sites. Average DNA methylation at new insertions in the Arabidopsis Ha-0 ecotype (middle), these same sites without TE insertion in the reference Col-0 ecotype (top), and the parental TEs in Ha-0 that produced each transposed TE (bottom). DNA methylation is split between cytosine sequence contexts (colors) and location at the insertion site (x-axis). Bar plots are shown on the left and a metaplot combining both the $5^{\prime}$ and $3^{\prime}$ TE ends and insertion sites is shown on the right. Error bars (left) and transparent colors (right) represent the $95 \% \mathrm{Cl}$. N/A not applicable

The major breakthrough of epiTEome is the ability to detect new TE insertion sites from a data source designed to detect cytosine DNA methylation. EpiTEome is efficient at TE insertion site detection, but also will identify the DNA methylation status of those sites. Even with both genome resequencing and MethylC-seq data in hand, the methylation of new TE insertion sites has not been investigated because both of these datasets are mapped to the reference genome, failing to identify non-reference TE positions. Our proof-of-principle results on one Arabidopsis mutant, one Arabidopsis ecotype, and between two maize inbred lines demonstrates that epiTEome can assay DNA methylation at new TE insertion sites in both simple and complex/repetitive genomes. We confirm DNA methylation recruitment to new TE insertion sites and a differential level of methylation between the nonreference and parental TE insertions. By generating the epiTEome program, we have provided the epigenetics community the resource to obtain more out of their data and assay two new features:TE insertion sites and their DNA methylation status, from existing or future MethylC-seq data.

\section{Conclusions}

EpiTEome provides, for the first time, the ability to detect new TE insertion events from new or existing MethylC-seq data. This program combines the analysis of new TE insertion sites with the analysis of TE DNA 
methylation into a single analysis, reducing the requirement to perform both genome resequencing and MethylC-seq on the same samples. EpiTEome enables new epigenomic investigation of previously overlooked non-reference $\mathrm{TE}$ insertion sites.

\section{Methods}

\section{Identification of new TE insertion sites}

All FASTQ files were trimmed for adapters and preprocessed to remove low quality reads using cutadapt [24] with the following parameters: -q $30-\max -\mathrm{n} 0$. Trimmed FASTQ reads were then mapped to a reference genome using the MethylC-Seq mapping program Bismark [14] using the following parameters: -bowtie2 -ambiguous -unmapped -R 10 -score_min $\mathrm{L}, 0,-0.6-\mathrm{N}$ 1. Identification of new TE insertion sites was performed using epiTEome (Fig. 1a). As output, epiTEome provides a file that contains the coordinates of the non-reference TE insertion sites, the type of mobile TEs, and the parental TE copy. In addition, epiTEome also outputs the methylation level at the edge of the non-reference TE and flanking insertion site.

\section{Simulated data}

For the analysis in Fig. 2a and b, 14 different types of TEs were inserted into a total of 84 loci on Arabidopsis chromosome 2. Three different TE insertion contexts (genic, intergenic, and TE) were chosen for each of the 84 neo-insertion sites, generating a synthetic chromosome 2 FASTA sequence. Among the three different TE insertion contexts, TE insertion sites were randomly chosen using bedtools shuffle and TEs were inserted using the custom Perl script insertTEsintoFasta.pl (available as part of epiTEome software package). We next in silico bisulfite-converted DNA sequencing reads with Sherman [25], using the rates of $20 \% \mathrm{CG}$ and $3 \% \mathrm{CH}$, which represent the average methylation level on Arabidopsis chromosome 2. In silico sequencing reads were produced using Sherman with a read length of $85 \mathrm{nt}$, a 20x genome coverage, and no SNPs. All three programs were launched on either the non-bisulfite-converted reads (TEPID and READSPLITER) or bisulfite-converted reads (epiTEome) and the outputted coordinates of TE neo-insertion sites were compared with the reference using bedmap [26]. This comparison allowed us to estimate the accuracy of each program by calculating the sensitivity $(\mathrm{TP} /(\mathrm{TP}+$ $\mathrm{FN})$ ) and the FDR $(\mathrm{FP} /(\mathrm{TP}+\mathrm{FP}))$. The same procedure was reproduced for the analysis in Fig. 2c using only epiTEome, 84 random insertion sites, and by altering independently the variables: depth of coverage; read length; number of SNPs; and percentage of methylation. A similar procedure was performed for Fig. $4 \mathrm{a}-\mathrm{c}$ :
LTR retrotransposons and DNA transposons were selected from TE families having a low (1-10), medium (11-100), and high (>101) copy number of TEs. Four hundred TEs were randomly inserted within three different TE insertion contexts (genic, intergenic, and TE) of maize chromosome 1 or rice chromosome 1 .

\section{EpiTEome analysis of the $\mathrm{Ha}-\mathrm{O}$ accession}

Coordinates of new TE insertion sites from the Arabidopsis ecotype Ha-0 identified by either SPLITREADER and TEPID were downloaded from [12] and [13]. Comparison of the new TE insertion site coordinates predicted by epiTEome with the two other methods was performed using bedmap. The proportional Venn diagram in Fig. 3a was produced using eulerAPE [27]. Validation of new TE insertion sites detected specifically by epiTEome was performed by calculating the un-split read coverage at each new TE insertion site (+/- $80 \mathrm{bp})$. In Fig. 3e, the unsplit read coverage standard score at the insertion site is clustered using the Ward's method of hierarchical cluster analysis (R package ggdendro).

\section{Analysis of non-reference TE insertions in maize}

The coordinates of new TE insertion sites were identified by epiTEome by aligning 100 bp bisulfite-converted reads to the maize reference genome (version 3) and corresponding version 3 annotation [28]. EpiTEome displayed enhanced sensitivity using the improved version 4 B73 reference sequence and annotation (unpublished data).

\section{Analysis of DNA methylation level}

DNA methylation level was assayed in each of the three cytosine contexts (CG, $\mathrm{CHG}$, and $\mathrm{CHH}$ ) at the flanking DNA surrounding the new TE insertion site and at both edges of the newly inserted TE. Only MethylC-seq discordant split-reads identified by epiTEome were used to calculate this methylation level. Meta-analysis was performed by averaging methylation levels at the $5^{\prime}$ and $3^{\prime}$ of the new insertion site within 10 nt windows. For any given window, the variation in methylation across all elements was used to calculate the 95\% CI.

\section{Availablility and Requirements}

EpiTEome is freely available at https:/github.com/ jdaron/epiTEome under a GNU General Public License and the source code has been deposited at Zenodo (doi: 10.5281/zenodo.495189). The general procedure by which epiTEome functions is described in Fig. 1 and in more detail within the README file distributed with the software package. EpiTEome requires the following list of dependencies: Perl, BioPerl, samtools,bedtools, ngsutils, and segemehl. 


\section{Acknowledgements}

The authors thank Kaushik Panda for his comments on this work.

\section{Funding}

This work is supported by the Ohio Supercomputer Center and the U.S. National Science Foundation grant MCB-1252370 to RKS.

\section{Availability of data and materials}

MethylC-seq data for Arabidopsis thaliana accessions were obtained from the NCBI SRA archive GSE43857 [17]. MethylC-Seq data from the ddm 1 mutant are from GSE79746 [29]. MethylC-seq data for the maize inbred lines were obtained from the NCBI SRA archive SRP048795 [21].

EpiTEome is freely available at https://github.com/jdaron/epiTEome under a GNU General Public License and the source code has been deposited at Zenodo (doi:10.5281/zenodo.495189). The general procedure by which epiTEome functions is described in Fig. 1 and in more detail within the README file distributed with the software package.

\section{Authors' contributions}

RKS contributed research funding. JD generated and tested epiTEome and analyzed the results. RKS and JD identified the initial research direction and co-wrote the paper. Both authors read and approved the final manuscript.

\section{Competing interests}

The authors declare that they have no competing interests.

\section{Ethics approval and consent to participate}

N/A

\section{Publisher's Note}

Springer Nature remains neutral with regard to jurisdictional claims in published maps and institutional affiliations.

\section{Received: 31 January 2017 Accepted: 5 May 2017}

Published online: 12 May 2017

\section{References}

1. Kidwell MG, Lisch D. Transposable elements as sources of variation in animals and plants. Proc Natl Acad Sci U S A. 1997:94:7704-11.

2. Hancks DC, Kazazian HH. Roles for retrotransposon insertions in human disease. Mob DNA. 2016;7:9.

3. Levin HL, Moran JV. Dynamic interactions between transposable elements and their hosts. Nat Rev Genet. 2011:12:615-27.

4. Wicker T, Sabot F, Hua-Van A, Bennetzen JL, Capy P, Chalhoub B, et al. A unified classification system for eukaryotic transposable elements. Nat Rev Genet. 2007;8:973-82.

5. Daron J, Glover N, Pingault L, Theil S, Jamilloux V, Paux E, et al. Organization and evolution of transposable elements along the bread wheat chromosome 3B. Genome Biol. 2014;15:546.

6. Kidwell MG. Transposable elements and the evolution of genome size in eukaryotes. Genetica. 2002;115:49-63.

7. Zhang J, Yu C, Krishnaswamy L, Peterson T. Transposable elements as catalysts for chromosome rearrangements. Methods Mol Biol. 2011;701:315-26.

8. Fultz D, Choudury SG, Slotkin RK. Silencing of active transposable elements in plants. Curr Opin Plant Biol. 2015;27:67-76.

9. Urich MA, Nery JR, Lister R, Schmitz RJ, Ecker JR. MethylC-seq library preparation for base-resolution whole-genome bisulfite sequencing. Nat Protoc. 2015:10:475-83.

10. Jiang N, Bao Z, Zhang X, Hirochika H, Eddy SR, McCouch SR, et al. An active DNA transposon family in rice. Nature. 2003:421:163-7.

11. Ewing AD. Transposable element detection from whole genome sequence data. Mob DNA. 2015:6:164

12. Quadrana L, Bortolini Silveira A, Mayhew GF, LeBlanc C, Martienssen RA, Jeddeloh JA, et al. The Arabidopsis thaliana mobilome and its impact at the species level. Elife. 2016:5:e15716.

13. Stuart T, Eichten S, Cahn J, Karpievitch Y, Borevitz J, Lister R. Population scale mapping of transposable element diversity reveals links to gene regulation and epigenomic variation. Elife. 2016;5:e20777.

14. Krueger $F$, Andrews SR. Bismark: a flexible aligner and methylation caller for Bisulfite-Seq applications. Bioinformatics. 2011;27:1571-2.
15. Otto C, Stadler PF, Hoffmann S. Fast and sensitive mapping of bisulfitetreated sequencing data. Bioinformatics. 2012;28:1698-704.

16. Cao J, Schneeberger K, Ossowski S, Günther T, Bender S, Fitz J, et al. Wholegenome sequencing of multiple Arabidopsis thaliana populations. Nat Genet. 2011;43:956-63.

17. Kawakatsu T, Huang S-SC, Jupe F, Sasaki E, Schmitz RJ, Urich MA, et al. Epigenomic diversity in a global collection of Arabidopsis thaliana accessions. Mol Cell. 2016;166:492-505.

18. 1001 Genomes Consortium. 1,135 genomes reveal the global pattern of polymorphism in Arabidopsis thaliana. Cell. 2016;166:481-91.

19. Schnable PS, Ware D, Fulton RS, Stein JC, Wei F, Pasternak S, et al. The B73 maize genome: complexity, diversity, and dynamics. Science. 2009:326:1112-5.

20. International Rice Genome Sequencing Project. The map-based sequence of the rice genome. Nature. 2005;436:793-800.

21. Li Q, Eichten SR, Hermanson PJ, Zaunbrecher VM, Song J, Wendt J, et al. Genetic perturbation of the maize methylome. Plant Cell. 2014;26:4602-16.

22. Ahmed I, Sarazin A, Bowler C, Colot V, Quesneville H. Genome-wide evidence for local DNA methylation spreading from small RNA-targeted sequences in Arabidopsis. Nucleic Acids Res. 2011;39:6919-31.

23. SanMiguel P, Tikhonov A, Jin YK, Motchoulskaia N, Zakharov D, MelakeBerhan A, et al. Nested retrotransposons in the intergenic regions of the maize genome. Science. 1996:274:765-8.

24. Martin M. Cutadapt removes adapter sequences from high-throughput sequencing reads. EMBnet J. 2011;17:10-2.

25. Krueger F. Sherman - bisulfite-treated Read FastQ Simulator. 2014. https:// www.bioinformatics.babraham.ac.uk/projects/sherman.

26. Neph S, Kuehn MS, Reynolds AP, Haugen E, Thurman RE, Johnson AK, et al. BEDOPS: high-performance genomic feature operations. Bioinformatics. 2012;28:1919-20.

27. Micallef L, Rodgers P. eulerAPE: drawing area-proportional 3-Venn diagrams using ellipses. PLoS One. 2014;9:e101717.

28. Law M, Childs KL, Campbell MS, Stein JC, Olson AJ, Holt C, et al. Automated update, revision, and quality control of the maize genome annotations using MAKER-P improves the B73 RefGen_v3 gene models and identifies new genes. Plant Physiol. 2014;167:25-39.

29. Panda K, Ji L, Neumann DA, Daron J, Schmitz RJ, Slotkin RK. Full-length autonomous transposable elements are preferentially targeted by expression-dependent forms of RNA-directed DNA methylation. Genome Biol. 2016:17:100

30. Miura A, Yonebayashi S, Watanabe K, Toyama T, Shimada H, Kakutani T. Mobilization of transposons by a mutation abolishing full DNA methylation in Arabidopsis. Nature. 2001:411:212-4.

\section{Submit your next manuscript to BioMed Central and we will help you at every step:}

- We accept pre-submission inquiries

- Our selector tool helps you to find the most relevant journal

- We provide round the clock customer support

- Convenient online submission

- Thorough peer review

- Inclusion in PubMed and all major indexing services

- Maximum visibility for your research

Submit your manuscript at www.biomedcentral.com/submit
Biomed Central 